Research Article

\title{
Reconstruction of a Robin Coefficient by a Predictor-Corrector Method
}

\author{
Yun-Jie Ma \\ School of Mathematics and Informational Science, Yantai University, Yantai 264005, China \\ Correspondence should be addressed to Yun-Jie Ma; mayunjie001@sina.com
}

Received 28 August 2015; Revised 18 November 2015; Accepted 22 November 2015

Academic Editor: Francisco Alhama

Copyright (C) 2015 Yun-Jie Ma. This is an open access article distributed under the Creative Commons Attribution License, which permits unrestricted use, distribution, and reproduction in any medium, provided the original work is properly cited.

The present paper is devoted to solving a nonlinear inverse problem of identifying a Robin coefficient from boundary temperature measurement. A numerical algorithm on the basis of the predictor-corrector method is designed to restore the approximate solution and the performance of the method is verified by simulating several examples. The convergence with respect to the amount of noise in the data is also investigated.

\section{Introduction}

Inverse heat conduction problems have important applications in many branches of engineering and science, including the identification of unknown source [1-3], identification of unknown heat transfer coefficients $[4,5]$, determination of boundary conditions [6], and thermal properties [7, 8]. It should be noted that the inverse problems are mostly ill posed and hence the accurate solutions of such problems are experimentally difficult to acquire.

In this paper, we investigate an inverse problem arising in transient convective heat transfer. It consists of estimating a heat transfer coefficient, also known as a Robin coefficient, which characterizes the contribution that an interface makes to the overall thermal resistance to the system. As the value of the coefficient is of significant practical interest in heat transfer engineering, various experimental techniques have been proposed. The conventional technique in thermal engineering employs the empirical correlations which represent curve fitting through experimental data in a limited range of flow-field parameters [9]. Some techniques based on characteristic colour changes of liquid crystal films at a given temperature [10], or on laser-induced fluorescence [11], rely in their interpretation on the analytical solution for a semiinfinite medium to determine the heat transfer coefficient at a point once the temperature history is obtained from the experiment. However, the impulsive change in boundary condition is difficult to achieve in the laboratory, and traditionally transient experiments have been performed to evaluate the heat transfer coefficient for steady-state processes so that such methods are difficult to use in truly time-dependent process where the heat transfer coefficient depends on time. Traditionally, partial boundary temperature and heat flux measurements are used as input to heat conduction models to extract the heat transfer coefficient values by solving a Cauchy linear inverse heat conduction problem. However, Cauchy data measurements may experience some practical difficulties, for example, in the case of high temperature hostile environments. Thus, in a more realistic model, we allow for the convective Robin boundary condition to be prescribed over the whole boundary and the linkage between the boundary temperature and heat flux be made through unknown heat transfer coefficient which varies with time $[12,13]$.

In recent decades, most of the theoretical and numerical results of the related problems are focused on the problem with Laplace equation [14]. Specifically, Alessandrini et al. [15] and Chaabane et al. [16] have obtained the stability estimates for the Robin coefficient. Fasino and Inglese [17, 18] have proposed some numerical algorithms for determining the Robin coefficient in a thin domain. Jin [19] has restored a Robin coefficient in the Laplace equation by using the conjugate gradient method. Some other relevant results can also be found in $[20,21]$. Later, the theoretical and numerical studies 
for restoring a Robin coefficient associated with the parabolic equations have appeared. For example, Onyango et al. [22] studied this problem using the boundary element method. Yang et al. [23] identified a Robin coefficient by a conjugate gradient method. Yan et al. [24] employed the Bayesian inference approach to recover the Robin coefficient in onedimensional transient inverse heat transfer problems. Jin and Lu [25] analyzed a regularization approach to the Robin inverse problem as well as its finite-element discretization. In this paper, we propose a high-efficiency iterative method based on the characteristics of the equation itself, that is, the predictor-corrector method, to solve the nonlinear inverse problem of identifying a time-dependent Robin coefficient. The traditional iterative method is a powerful tool for solving the nonlinear problem, but there may be some deficiencies in computation; for example, there will be a large amount of computation if the initial value is not appropriate. The predictor-corrector method is a combination of implicit algorithm with stability and accuracy and explicit algorithm with simplicity and thus is the most widely used method in the linear multistep methods. The method does not require either a priori information on the unknown solution or very accurate initial guess data, and it has been successfully applied to solve some inverse problems (see $[26,27]$ ).

Our paper is organized as follows. In Section 2, we formulate mathematically the identification problem of a Robin coefficient. In Section 3, the predictor-corrector method involving the finite difference method is employed to solve this problem. In Section 4, numerical results for several examples are presented to show the effectiveness of the suggested method. Finally, Section 5 ends this paper with a short conclusion.

\section{Mathematical Formulation of the Problem}

In this paper, we consider the following inverse heat conduction problem:

$$
\begin{aligned}
u_{t}-a^{2} u_{x x} & =0, \\
(x, t) \in Q:=(0, L) \times\left(0, t_{\max }\right), & \\
u(x, 0) & =\varphi(x), \quad x \in(0, L), \\
-\frac{\partial u}{\partial x}(0, t)+\sigma(t) u(0, t) & =g_{0}(t), \quad t \in\left(0, t_{\max }\right), \\
\frac{\partial u}{\partial x}(L, t)+\sigma(t) u(L, t) & =g_{1}(t), \quad t \in\left(0, t_{\max }\right),
\end{aligned}
$$

where $t_{\max }>0$ is an arbitrary fixed time of interest, $a$ is thermal diffusivity, $\varphi$ is a specified function of space representing the initial temperature, $g_{0}$, and $g_{1}$ are specified functions of time representing the heat flux, and $\sigma(t)$ is the Robin coefficient of energy exchange to be determined. It is well known that problem (1) is a well-posed direct problem if $\sigma(t)$ is given, and it has been studied extensively, while if $\sigma(t)$ is unknown, problem (1) is underdetermined; that is, it is not realistic to determine both a solution and a Robin coefficient just from the initial observation $\varphi$ and boundary value data $g_{0}, g_{1}$. Additional information, called "effect," is necessary to be measured in order to compensate for the unknown "causes" of the inverse problems. In this paper, the additional temperature measurement is given by

$$
u(0, t)=\psi(t), \quad t \in\left[0, t_{\max }\right]
$$

According to the result in $[22,28]$, the coefficient $\sigma(t)$ which satisfies (1) could be determined uniquely by condition (2).

Theorem 1 (see $[22,28])$. Suppose $\varphi(x) \in C^{1}(0, L) \cap C[0, L]$, $g_{0}(t), g_{1}(t), \psi(t) \in C\left[0, t_{\max }\right]$, and $|\psi(t)|>0$ for all $t \in$ $\left[0, t_{\max }\right]$; then, the solution pair $\sigma(t) \in C\left[0, t_{\max }\right]$ and $u(x, t) \in$ $C^{2,1}(Q)$ of the inverse problem (1) and (2) is unique.

In order to solve the inverse problem by using predictorcorrector method, we first discretize problem (1). Let $M$ and $J$ be the numbers of grid on the space and time domains, respectively, $h=\Delta x=L /(M-1), x_{i}=(i-1) h, i=$ $1,2, \ldots, M, \tau=\Delta t=t_{\max } /(J-1)$, and $t_{k}=(k-1) \tau, k=$ $1,2, \ldots, J$. Denote $u_{i}^{k}$ as the approximate value of $u\left(x_{i}, t_{k}\right)$. Then, for $k=1,2, \ldots, J-1$, the discrete form of (1) is as follows:

$$
\begin{gathered}
u_{i}^{k+1}-u_{i}^{k}-a^{2} r\left(u_{i+1}^{k+1}-2 u_{i}^{k+1}+u_{i-1}^{k+1}\right)=0, \\
i=2,3, \ldots, M-1, \\
u_{i}^{1}=\varphi_{i}, \\
i=1,2, \ldots, M, \\
-\left[\frac{u_{2}^{k+1}-u_{1}^{k+1}}{h}-\frac{h\left(u_{1}^{k+1}-u_{1}^{k}\right)}{2 a^{2} \tau}\right]+\sigma^{k+1} u_{1}^{k+1}=g_{0}^{k+1}, \\
\frac{u_{M}^{k+1}-u_{M-1}^{k+1}}{h}+\frac{h\left(u_{M}^{k+1}-u_{M}^{k}\right)}{2 a^{2} \tau}+\sigma^{k+1} u_{M}^{k+1}=g_{1}^{k+1},
\end{gathered}
$$

where $r=\tau / h^{2}$. The corresponding matrix notation could be written as

$$
A^{k+1} U^{k+1}=U^{k}+G^{k+1},
$$

where

$$
\begin{aligned}
U^{k} & =\left[u_{1}^{k}, u_{2}^{k}, \ldots, u_{M}^{k}\right]^{T}, \\
G^{k} & =\frac{2 \tau}{h}\left[g_{0}^{k}, 0, \ldots, 0, g_{1}^{k}\right]^{T},
\end{aligned}
$$




$$
A^{k+1}=\left(\begin{array}{cccc}
1+2 a^{2} r+\frac{2 a^{2} \tau}{h} \sigma^{k+1} & -2 a^{2} r & & \\
-a^{2} r & 1+2 a^{2} r & -a^{2} r & \\
& \ddots & \ddots & \\
& -a^{2} r & 1+2 a^{2} r & -a^{2} r \\
& & -2 a^{2} r & 1+2 a^{2} r+\frac{2 a^{2} \tau}{h} \sigma^{k+1}
\end{array}\right) .
$$

For given $\sigma(t)$, from classical textbooks, we know that the implicit difference scheme is convergent for any $r>0$.

\section{Predictor-Corrector Method}

Suppose the conditions in Theorem 1 hold. From the boundary condition in (1) and measurement data (2), the Robin coefficient could be written as

$$
\sigma(t)=\frac{g_{0}(t)+u_{x}(0, t)}{\psi(t)}
$$

at $t=t_{k}$,

$$
\sigma^{k}=\sigma\left(t_{k}\right)=\frac{g_{0}\left(t_{k}\right)+u_{x}\left(0, t_{k}\right)}{\psi\left(t_{k}\right)} .
$$

Thus, for initial guess data of $\sigma^{k}$, which is denoted by $\sigma^{k(0)}$, we would obtain the first-order update $\sigma^{k(1)}$ of $\sigma^{k}$ from the iterative equation (7). Due to

$$
\sigma^{1}=\frac{g_{0}\left(t_{1}\right)+\varphi^{\prime}\left(x_{1}\right)}{\psi\left(t_{1}\right)}
$$

it provides a reasonable initial value for $\sigma(t)$ at $t=t_{1}$. In the process of computing $\sigma^{k+1}$, the initial guess data of $\sigma^{k+1}$ are selected to be $\sigma^{k+1(0)}=\sigma^{k}, k=1,2, \ldots, J-1$.

Assume that we have obtained the value of $\sigma^{1}, \sigma^{2}, \ldots, \sigma^{k}$; the process of solving $\sigma^{k+1}$ and $U^{k+1}$ is as follows:

(1) Choose $\sigma^{k+1(0)}$. For simplicity, we choose $\sigma^{k+1(0)}=\sigma^{k}$, $k \leq J-1$.

(2) By using the implicit difference scheme, we solve problem (1) with $\sigma=\sigma^{k+1(0)}$ to approximate $u_{x}\left(x_{1}, t_{k+1} ; \sigma^{k+1(0)}\right)$, which is computed by formula $\left(u_{2}^{k+1}-u_{1}^{k+1}\right) / h-\left(h / 2 a^{2} \tau\right)\left(u_{1}^{k+1}-u_{1}^{k}\right)$. Thus, the firstorder update could be obtained as follows:

$$
\sigma^{k+1(1)}=\frac{g_{0}\left(t_{k+1}\right)+u_{x}\left(x_{1}, t_{k+1} ; \sigma^{k+1(0)}\right)}{\psi\left(t_{k+1}\right)} .
$$

(3) Choose an arbitrary small positive constant $\varepsilon$ to be the error bound. Compute $\left|\sigma^{k+1(1)}-\sigma^{k+1(0)}\right|$ and compare it with $\varepsilon$.
If

$$
\left|\sigma^{k+1(1)}-\sigma^{k+1(0)}\right|<\varepsilon
$$

then the iteration stops, and we take $\sigma^{k+1}=\sigma^{k+1(1)}$. If

$$
\left|\sigma^{k+1(1)}-\sigma^{k+1(0)}\right| \geq \varepsilon
$$

then $\sigma^{k+1(1)}$ is selected to be new initial guess data, and go to Step (1); repeat the above procedure until convergence is achieved.

\section{Numerical Results and Discussion}

In this section, we present the numerical results obtained by using the predictor-corrector method described in Section 3 for several examples. In our computation, we always set $L=1$, $t_{\max }=1$.

Note that, in practical situations, the data $\psi$ is measured and inevitably contaminated by noise. Thus, in our examples, we will replace the exact data $\psi$ by

$$
\Psi_{\delta}=\Psi(1+\delta \operatorname{rand}(\operatorname{size}(\Psi))),
$$

where $\Psi$ is the discrete form of $\psi$ and $\operatorname{rand}(\cdot)$ generates arrays of uniform random numbers. To test the accuracy of the computed approximations, we use the relative root mean square error $e_{r}$ which is defined as

$$
e_{r}=\frac{\sqrt{\sum_{k=1}^{J}\left(\sigma\left(t_{k}\right)-\sigma^{k}\right)^{2}}}{\sqrt{\sum_{k=1}^{J} \sigma^{2}\left(t_{k}\right)}} .
$$

We consider the following four examples.

Example 1 $\left(a=1, \varphi(x)=x^{2}+1, g_{0}(t)=(t+1)(2 t+1)\right.$, $\left.g_{1}(t)=2+2(t+1)^{2}\right)$. The Robin coefficient $\sigma(t)$ is a smooth function:

$$
\sigma(t)=1+t, \quad 0 \leq t \leq 1
$$

For this example, the direct problem (1) has the analytical solution

$$
u(x, t)=x^{2}+2 t+1
$$




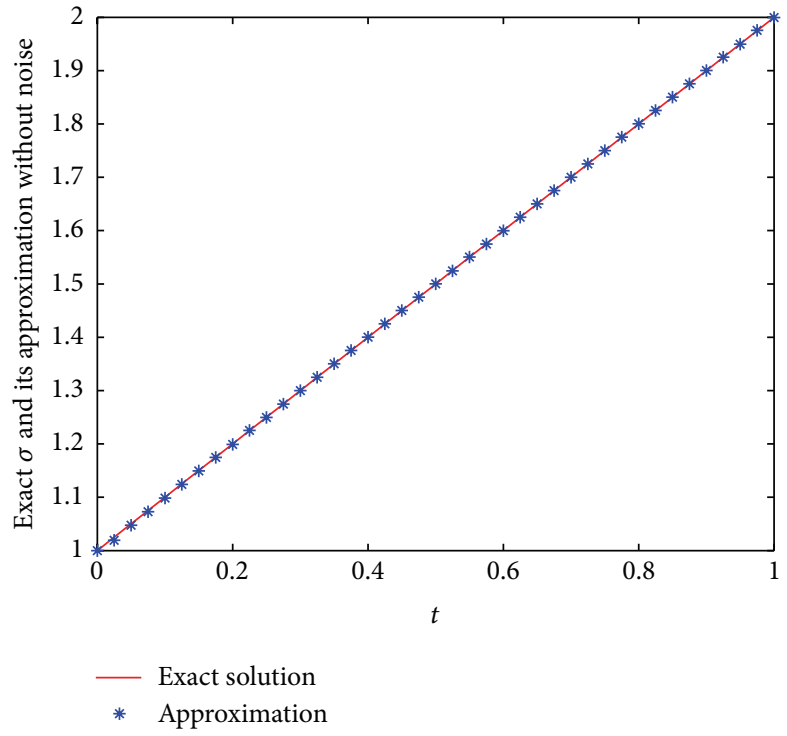

(a)

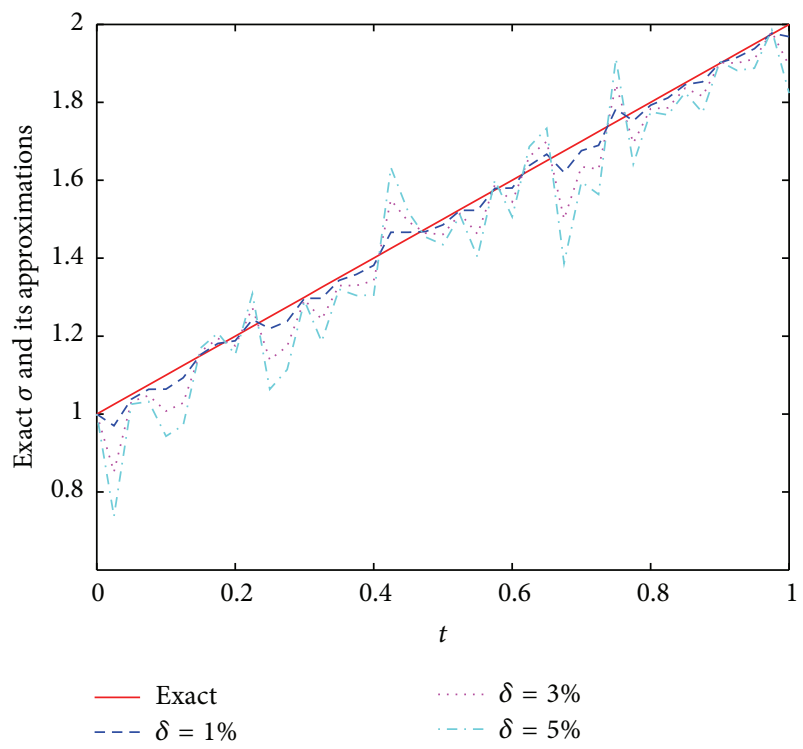

(b)

Figure 1: The exact solution $\sigma(t)$ and its approximations $\sigma^{k}$ with (a) $\delta=0 \%$ and (b) $\delta=1 \%, 3 \%$, and $5 \%$ for Example 1 .

Example $2\left(a=1, \varphi(x)=x^{2}+1, g_{0}(t)=(1.5+\right.$ $\left.\sin (2 \pi t))(2 t+1), g_{1}(t)=2+2(t+1)(1.5+\sin (2 \pi t))\right)$. The Robin coefficient $\sigma(t)$ is a smooth function:

$$
\sigma(t)=1.5+\sin (2 \pi t), \quad 0 \leq t \leq 1 .
$$

For this example, the analytical solution of direct problem (1) is the same as Example 1. For these two examples, the numbers of grid on the space-time domain are taken to be $M=41, J=41$. Some similar examples also have been investigated previously by Onyango et al. [22] and Yang et al. [23] by using the boundary element method and a conjugate gradient method, respectively.

In the following, we consider two examples where there are no analytical solutions in a more realistic context. Assume that $a=\sqrt{10}, \varphi(x)=1, g_{0}(t)=2000$, and $g_{1}(t)=0$. For these cases, both $M$ and $J$ are taken to be 81 .

Example 3. The Robin coefficient $\sigma(t)$ is a nonsmooth but continuous function:

$$
\sigma(t)= \begin{cases}1+2 t, & 0 \leq t \leq 0.5 \\ 3-2 t, & 0.5<t \leq 1\end{cases}
$$

Example 4. The Robin coefficient $\sigma(t)$ is a discontinuous function:

$$
\sigma(t)= \begin{cases}2, & 0.25<t<0.75 \\ 1, & \text { otherwise }\end{cases}
$$

As the direct problem (1) does not have an analytical solution for Examples 3 and 4, the boundary temperature $\psi$ should be obtained by solving the direct problem. In order to avoid the notorious "inverse crime," we will solve the direct problem on a finer mesh.
TABLE 1: Number of iterations on every discrete point for Example 1 with $\delta=3 \%$.

\begin{tabular}{lccccccccccccc}
\hline$t$ & $t_{2}$ & $t_{3}$ & $t_{4}$ & $t_{5}$ & $t_{6}$ & $t_{7}$ & $t_{8}$ & $t_{9}$ & $t_{10}$ & $t_{11}$ & $t_{12}$ & $t_{13}$ & $t_{14}$ \\
$k$ & 6 & 18 & 17 & 3 & 21 & 9 & 10 & 20 & 8 & 15 & 9 & 19 & 14 \\
\hline$t_{15}$ & $t_{16}$ & $t_{17}$ & $t_{18}$ & $t_{19}$ & $t_{20}$ & $t_{21}$ & $t_{22}$ & $t_{23}$ & $t_{24}$ & $t_{25}$ & $t_{26}$ & $t_{27}$ & $t_{28}$ \\
15 & 10 & 1 & 13 & 4 & 12 & 14 & 13 & 13 & 13 & 3 & 4 & 12 & 13 \\
\hline$t_{29}$ & $t_{30}$ & $t_{31}$ & $t_{32}$ & $t_{33}$ & $t_{34}$ & $t_{35}$ & $t_{36}$ & $t_{37}$ & $t_{38}$ & $t_{39}$ & $t_{40}$ & $t_{41}$ & \\
9 & 12 & 13 & 5 & 7 & 7 & 14 & 7 & 10 & 12 & 8 & 10 & 12 & \\
\hline
\end{tabular}

TABLE 2: The relative errors for four examples with various noise in the data.

\begin{tabular}{lcccc}
\hline$\delta$ & $\begin{array}{c}e_{r} \\
\text { (Example 1) }\end{array}$ & $\begin{array}{c}e_{r} \\
\text { (Example 2) }\end{array}$ & $\begin{array}{c}e_{r} \\
\text { (Example 3) }\end{array}$ & $\begin{array}{c}e_{r} \\
\text { (Example 4) }\end{array}$ \\
\hline$\delta=0 \%$ & 0.0007 & 0.0022 & 0.0194 & 0.0248 \\
$\delta=1 \%$ & 0.0152 & 0.0146 & 0.0230 & 0.0273 \\
$\delta=3 \%$ & 0.0456 & 0.0438 & 0.0372 & 0.0395 \\
$\delta=5 \%$ & 0.0757 & 0.0721 & 0.0541 & 0.0554 \\
\hline
\end{tabular}

For Example 1, the numerical solution for $\sigma(t)$ with exact data is given in Figure 1(a), and Figure 1(b) is the comparison of exact solution and its approximations with different noise in the measurement data. From Figures 1(a) and 1(b), it is observed that the numerical effect is acceptable, and the smaller the noise $\delta$, the more accurate the approximations. The accuracies of the approximations with various noise are presented in Table 2.

Table 1 lists the number of iterations on every discrete point $t_{k}(k=2,3, \ldots, J)$ when solving $\sigma^{k}$ for Example 1 with $\delta=3 \%$ in the data. It is worth noting that we would achieve the accuracy requirement after several, at most 


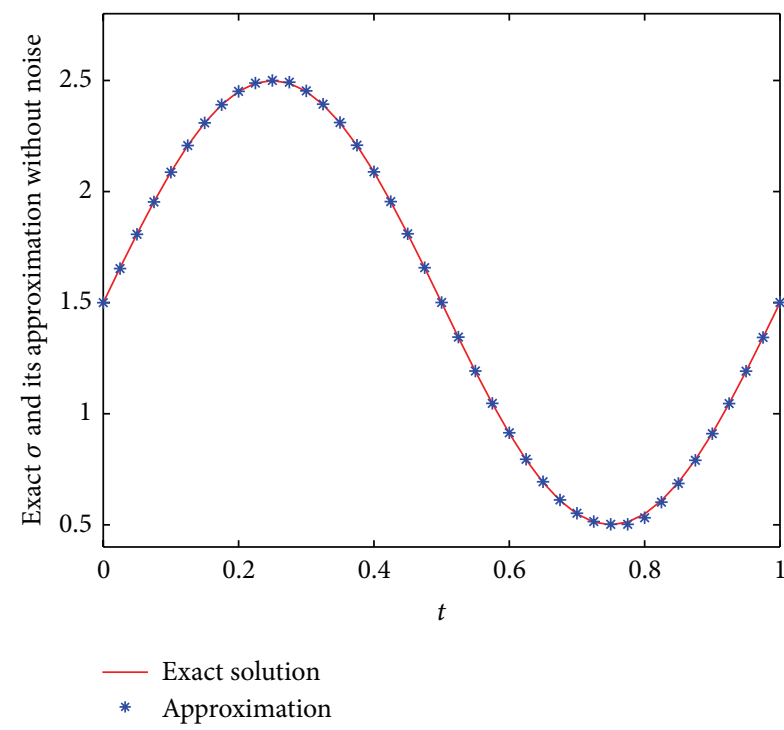

(a)

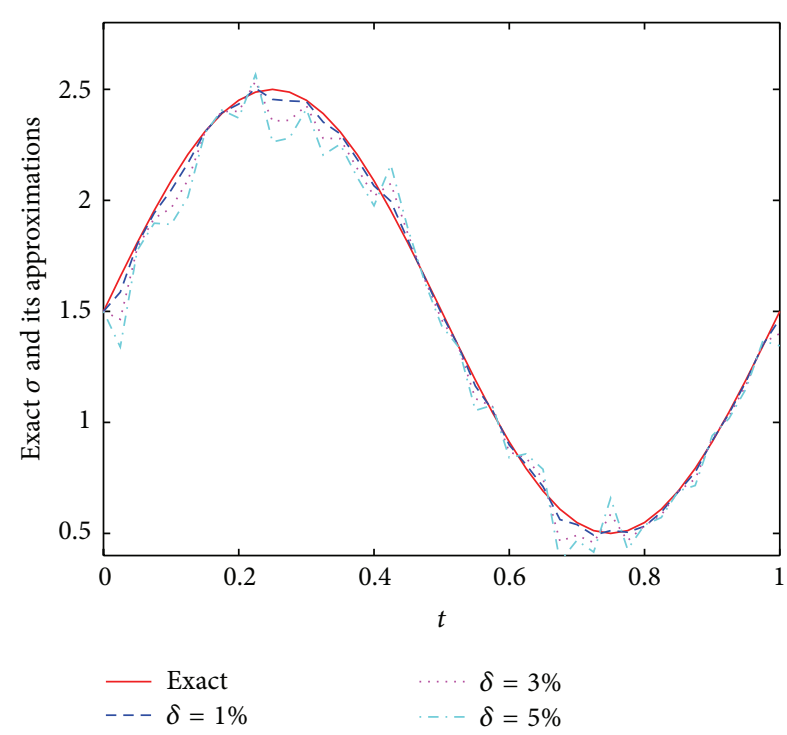

(b)

Figure 2: The exact solution $\sigma(t)$ and its approximations $\sigma^{k}$ with (a) $\delta=0 \%$ and (b) $\delta=1 \%, 3 \%$, and $5 \%$ for Example 2.

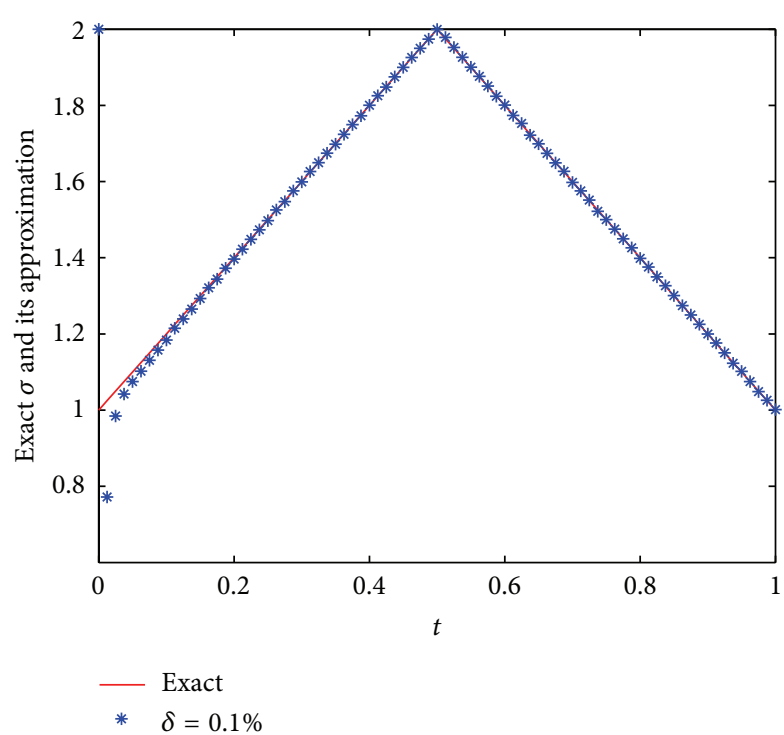

(a)

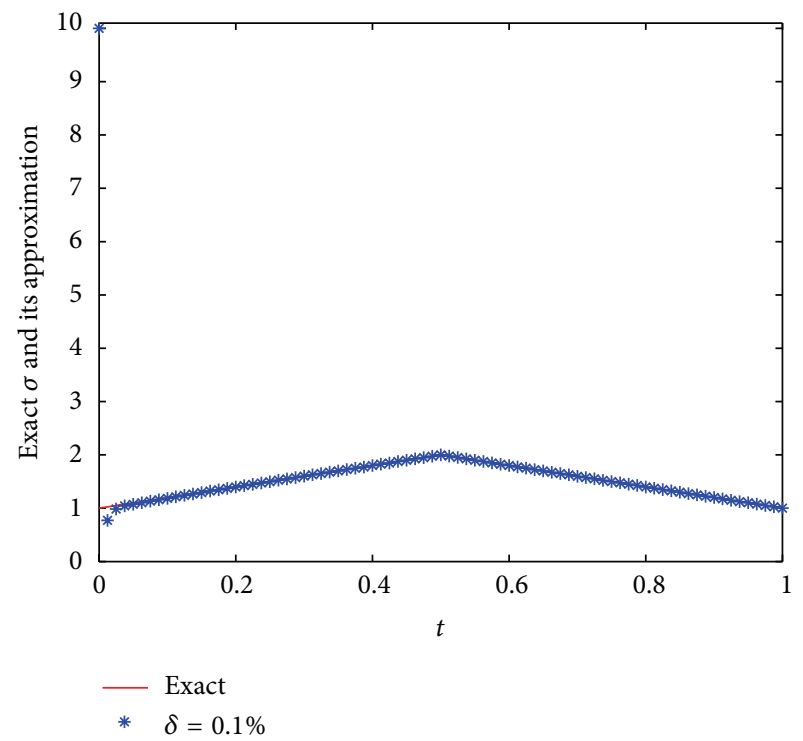

(b)

FIGURE 3: Exact $\sigma(t)$ and its approximations for (a) $\sigma^{1}=2$ and (b) $\sigma^{1}=10$ with $\delta=0.1 \%$ for Example 3 .

a dozen of, iterations. This table shows the high efficiency of the predictor-corrector method.

Figures 2(a) and 2(b) give the comparison of exact Robin coefficient with its approximations with various amount of noise in the data. From Figure 2(b), we see that, with up to $\delta=5 \%$, the approximate solution of $\sigma(t)$ is in good agreement with the exact solution. The accuracies of the approximations with various noise are also presented in Table 2.

As the initial guess data of $\sigma$ at $t=t_{1}$ is usually difficult to be obtained in practical application, we test the impact of initial guess data on the numerical result for Examples 3 and 4, respectively. $\sigma^{1}$ is taken to be 2 in Figures 3(a) and 4(a), and $\sigma^{1}$ is taken to be 10 in Figures 3(b) and 4(b). From these figures, it is observed that the approximations converge quickly to exact solutions for discrete points $t_{k}(k>2)$, and this shows that the predictor-corrector method is not sensitive to the initial guess.

Figures 5 and 6 show the numerical results for nonsmooth and discontinuous Robin coefficient, respectively. From these figures, we see that the approximations of Robin coefficient are in good agreement with the exact one. Generally speaking, problems with nonsmooth solutions present 


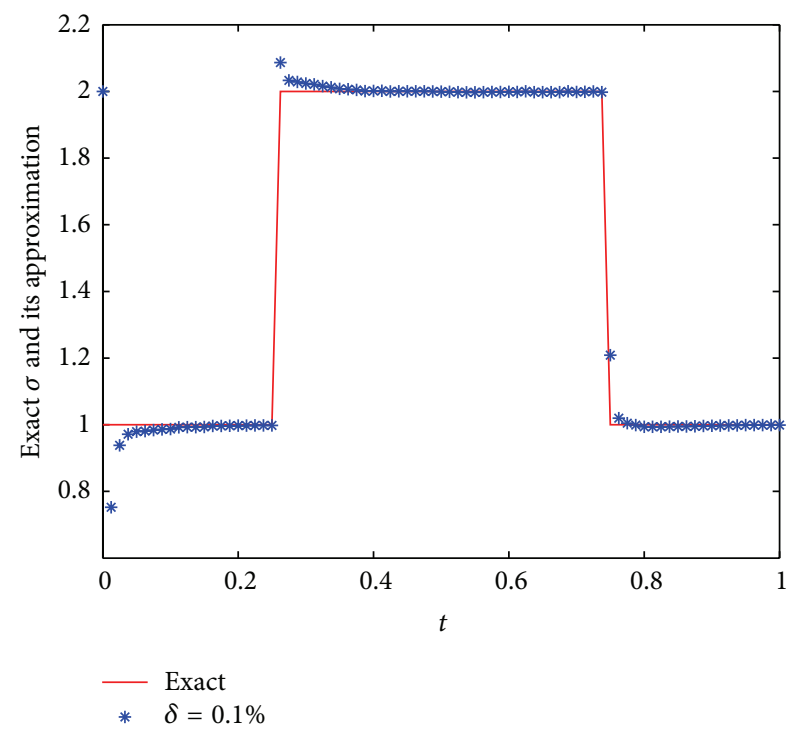

(a)

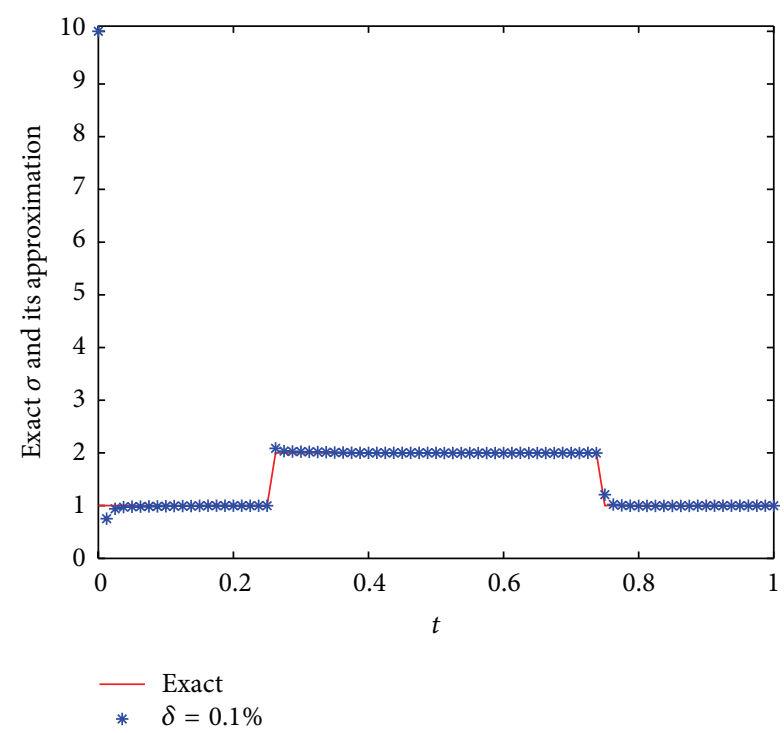

(b)

FIGURE 4: Exact $\sigma(t)$ and its approximations for (a) $\sigma^{1}=2$ and (b) $\sigma^{1}=10$ with $\delta=0.1 \%$ for Example 4 .

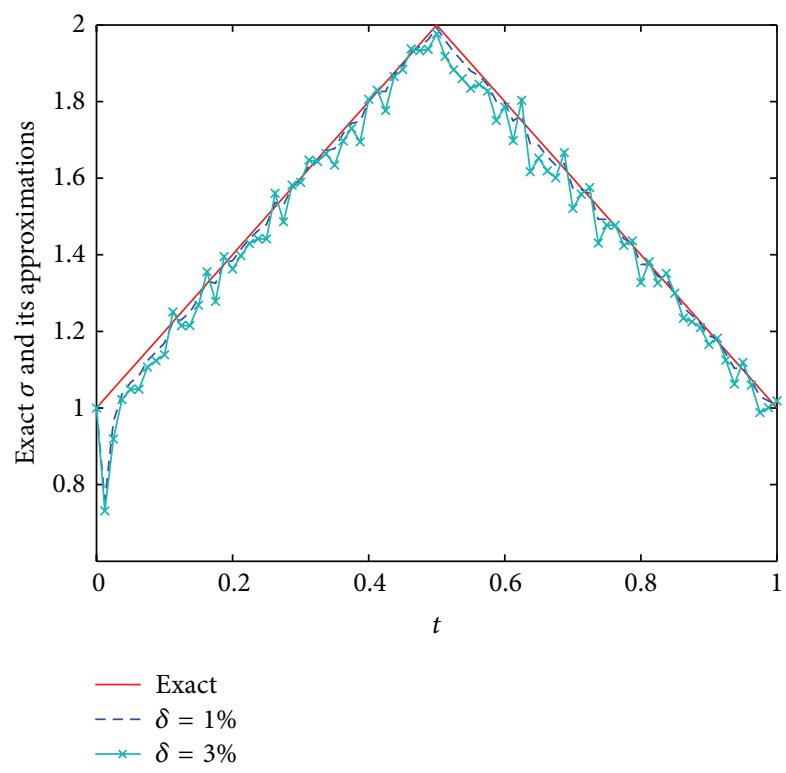

FIGURE 5: The exact solution $\sigma(t)$ and its approximations $\sigma^{k}$ with $\delta=1 \%$ and $3 \%$ for Example 3 .

major challenges in numerical computation aspect, and it is often difficult to obtain accurate approximations, while the predictor-corrector method works well for this case. From Table 2, the relative errors for Examples 3 and 4 are as small as the ones for Examples 1 and 2. This shows the effectiveness of the algorithm for nonsmooth and discontinuous Robin coefficient.

\section{Conclusion}

The inverse problem of restoring an unknown Robin coefficient in heat conduction problem is investigated by

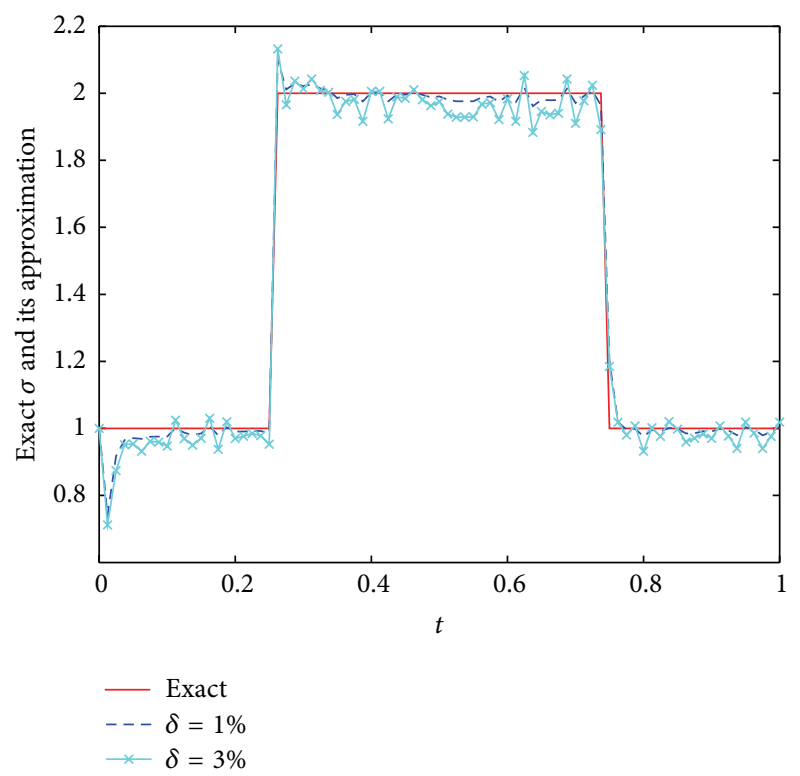

FIGURE 6: The exact solution $\sigma(t)$ and its approximations $\sigma^{k}$ with $\delta=1 \%$ and $3 \%$ for Example 4 .

employing the predictor-corrector method. The method is a combination of implicit algorithm with stability and explicit algorithm with simplicity. The numerical results for several examples show that the approximations can be restored very well by the suggested method.

\section{Conflict of Interests}

The author declares that there is no conflict of interests regarding the publication of this paper. 


\section{Acknowledgments}

The project is supported by the NNSF of China (nos. 11171136 and 11571295), the Tianyuan Fund for Mathematics of the NSF of China (no. 11326235), the Youth Foundation of NNSF of China (nos. 11301301 and 61403327), and the Natural Science Foundation of Shandong Province (no. BS2013SF027).

\section{References}

[1] J. R. Cannon, "Determination of an unknown heat source from overspecified boundary data," SIAM Journal on Numerical Analysis, vol. 5, pp. 275-286, 1968.

[2] F.-F. Dou and C.-L. Fu, "Determining an unknown source in the heat equation by a wavelet dual least squares method," Applied Mathematics Letters, vol. 22, no. 5, pp. 661-667, 2009.

[3] B. T. Johansson and D. Lesnic, "A variational method for identifying a spacewise-dependent heat source," IMA Journal of Applied Mathematics, vol. 72, no. 6, pp. 748-760, 2007.

[4] S. Chantasiriwan, "Inverse heat conduction problem of determining time-dependent heat transfer coefficient," International Journal of Heat and Mass Transfer, vol. 42, no. 23, pp. 4275-4285, 1999.

[5] E. Divo, A. J. Kassab, J. S. Kapat, and M.-K. Chyu, "Retrieval of multidimensional heat transfer coefficient distributions using an inverse BEM-based regularized algorithm: numerical and experimental results," Engineering Analysis with Boundary Elements, vol. 29, no. 2, pp. 150-160, 2005.

[6] D. Lesnic, L. Elliott, and D. B. Ingham, "Application of the boundary element method to inverse heat conduction problems," International Journal of Heat and Mass Transfer, vol. 39, no. 7, pp. 1503-1517, 1996.

[7] C.-H. Huang and Y. Jan-Yuan, "An inverse problem in simultaneously measuring temperature-dependent thermal conductivity and heat capacity," International Journal of Heat and Mass Transfer, vol. 38, no. 18, pp. 3433-3441, 1995.

[8] D. Lesnic, L. Elliott, and D. B. Ingham, "Identification of the thermal conductivity and heat capacity in unsteady nonlinear heat conduction problems using the boundary element method," Journal of Computational Physics, vol. 126, no. 2, pp. 410-420, 1996.

[9] F. M. White, Heat and Mass Transfer, Addison-Wesley, Reading, Mass, USA, 1988.

[10] S. A. Hippensteele, L. M. Russell, and F. S. Stepka, "Evaluation of a method for heat transfer measurements and thermal visualization using a composite of a heater element and liquid crystals," Journal of Heat Transfer, vol. 105, no. 1, pp. 184-189, 1983.

[11] D. J. Bizzak and M. K. Chyu, "Use of a laser-induced fluorescence thermal imaging system for local jet impingement heat transfer measurement," International Journal of Heat and Mass Transfer, vol. 38, no. 2, pp. 267-274, 1995.

[12] D. N. H'ao, P. X. Thanh, and D. Lesnic, "Determination of the heat transfer coefficients in transient heat conduction," Inverse Problems, vol. 29, no. 9, 2013.

[13] M. Slodička, D. Lesnic, and T. T. M. Onyango, "Determination of a time-dependent heat transfer coefficient in a nonlinear inverse heat conduction problem," Inverse Problems in Science and Engineering, vol. 18, no. 1, pp. 65-81, 2010.

[14] G. Inglese, "An inverse problem in corrosion detection," Inverse Problems, vol. 13, no. 4, pp. 977-994, 1997.
[15] G. Alessandrini, L. Del Piero, and L. Rondi, "Stable determination of corrosion by a single electrostatic boundary measurement," Inverse Problems, vol. 19, no. 4, pp. 973-984, 2003.

[16] S. Chaabane, I. Fellah, M. Jaoua, and J. Leblond, "Logarithmic stability estimates for a Robin coefficient in two-dimensional Laplace inverse problems," Inverse Problems, vol. 20, no. 1, pp. 47-59, 2004.

[17] D. Fasino and G. Inglese, "An inverse Robin problem for Laplace's equation: theoretical results and numerical methods," Inverse Problems, vol. 15, no. 1, pp. 41-48, 1999.

[18] D. Fasino and G. Inglese, "Discrete methods in the study of an inverse problem for Laplace's equation," IMA Journal of Numerical Analysis, vol. 19, no. 1, pp. 105-118, 1999.

[19] B. T. Jin, "Conjugate gradient method for the Robin inverse problem associated with the Laplace equation," International Journal for Numerical Methods in Engineering, vol. 71, no. 4, pp. 433-453, 2007.

[20] W. Fang and M. Lu, "A fast collocation method for an inverse boundary value problem," International Journal for Numerical Methods in Engineering, vol. 59, no. 12, pp. 1563-1585, 2004.

[21] F. Lin and W. Fang, "A linear integral equation approach to the Robin inverse problem," Inverse Problems, vol. 21, no. 5, pp. 1757-1772, 2005.

[22] T. T. M. Onyango, D. B. Ingham, and D. Lesnic, "Reconstruction of heat transfer coefficients using the boundary element method," Computers \& Mathematics with Applications, vol. 56, no. 1, pp. 114-126, 2008.

[23] F. L. Yang, L. Yan, and T. Wei, "The identification of a Robin coefficient by a conjugate gradient method," International Journal for Numerical Methods in Engineering, vol. 78, no. 7, pp. 800-816, 2009.

[24] L. Yan, F. L. Yang, and C. L. Fu, "A Bayesian inference approach to identify a Robin coefficient in one-dimensional parabolic problems," Journal of Computational and Applied Mathematics, vol. 231, no. 2, pp. 840-850, 2009.

[25] B. T. Jin and X. L. Lu, "Numerical identification of a Robin coefficient in parabolic problems," Mathematics of Computation, vol. 81, no. 279, pp. 1369-1398, 2012.

[26] D. S. Daoud, "Determination of the source parameter in a heat equation with a non-local boundary condition," Journal of Computational and Applied Mathematics, vol. 221, no. 1, pp. 261272, 2008.

[27] Z.-C. Deng and L. Yang, "An inverse problem of identifying the coefficient of first-order in a degenerate parabolic equation," Journal of Computational and Applied Mathematics, vol. 235, no. 15, pp. 4404-4417, 2011.

[28] A. B. Kostin and A. I. Prilepko, "Some problems of restoring the boundary condition for a prarbolic equation II," Differential Equations, vol. 32, no. 11, pp. 1515-1525, 1996. 


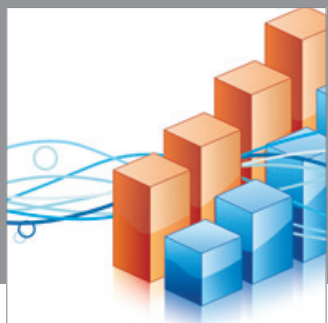

Advances in

Operations Research

mansans

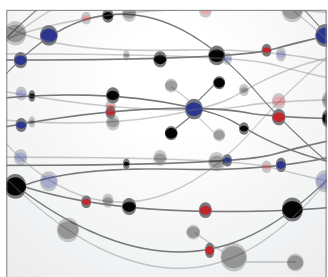

The Scientific World Journal
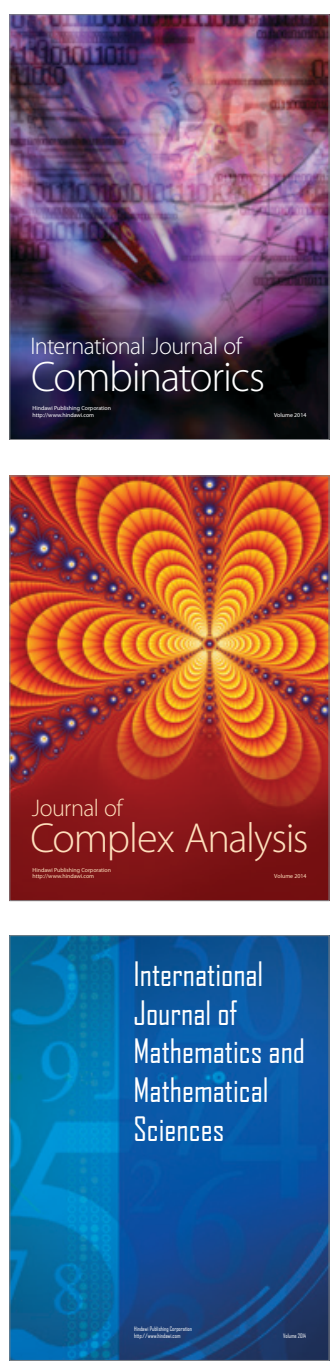
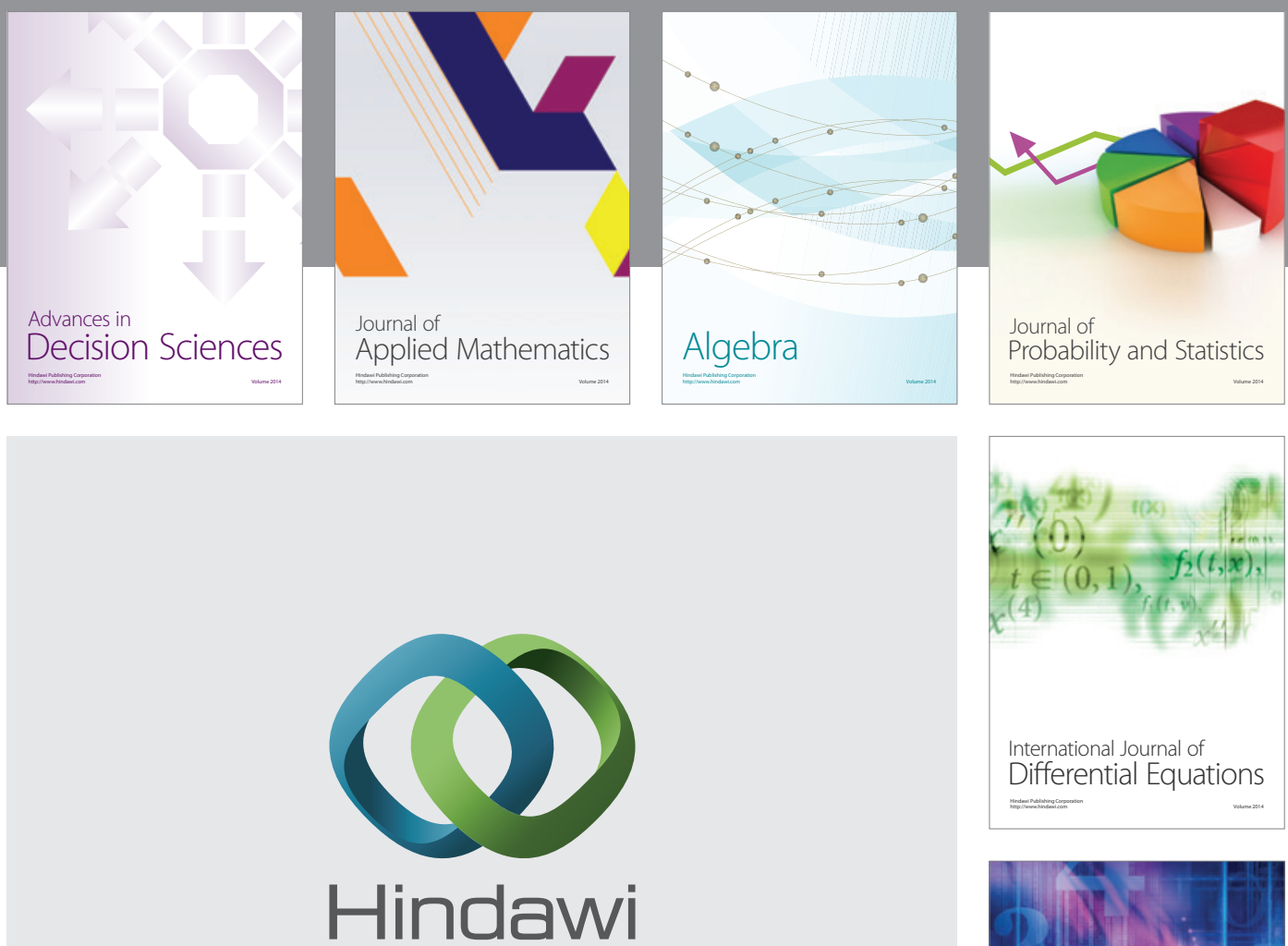

Submit your manuscripts at http://www.hindawi.com
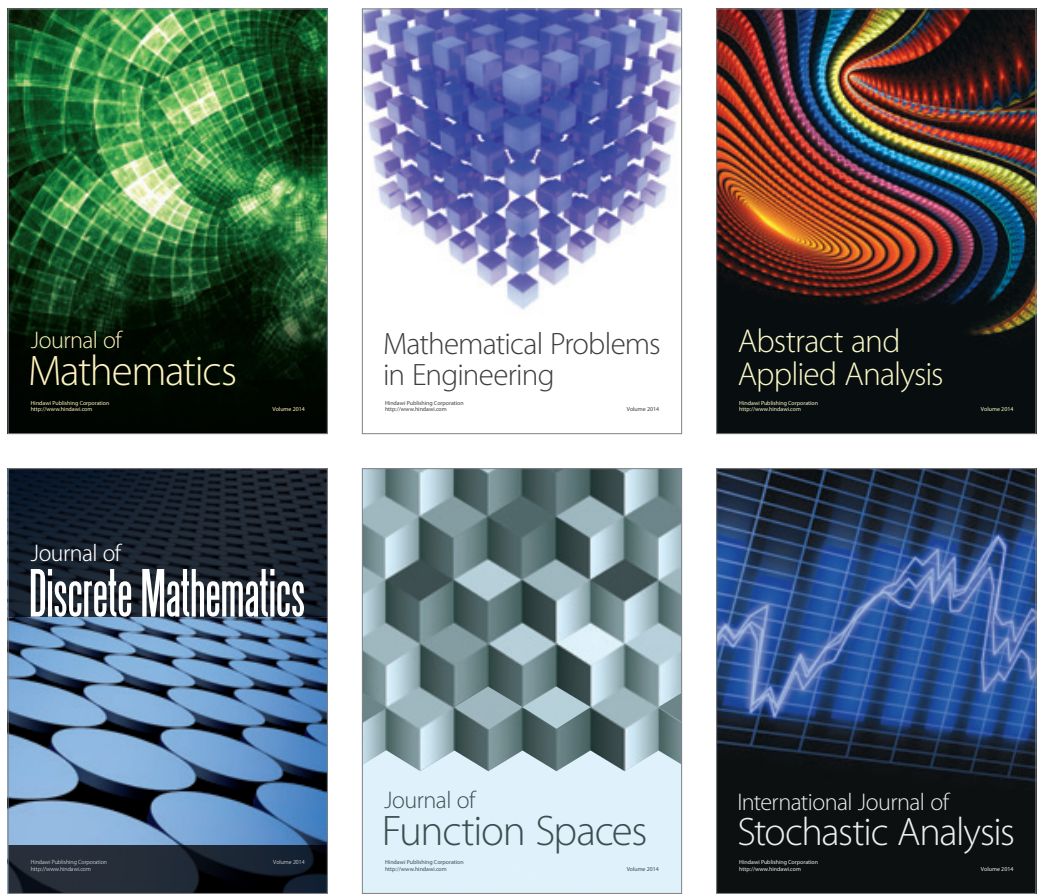

Journal of

Function Spaces

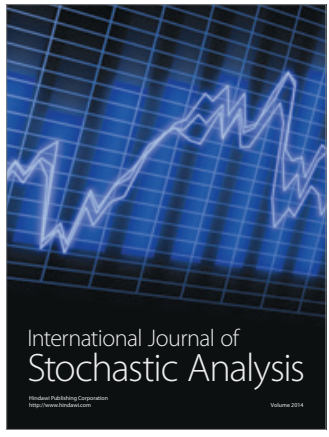

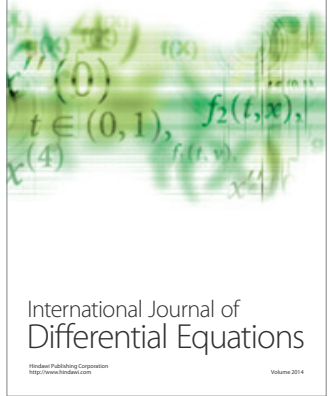
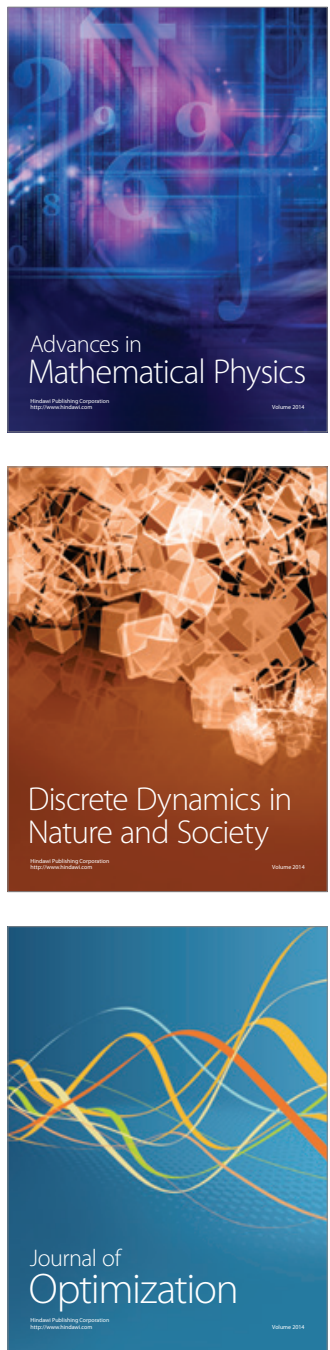